\title{
FPR2 enhances colorectal cancer progression by promoting EMT process
}

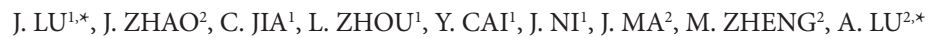 \\ ${ }^{1}$ Department of General Surgery, Affiliated Hangzhou First People's Hospital, Zhejiang University School of Medicine, Hangzhou, China; \\ ${ }^{2}$ Shanghai Minimally Invasive Surgery Center, Ruijin Hospital, Shanghai Jiao Tong University School of Medicine, Shanghai, China
}

*Correspondence: adams_lu66@126.com; 13788989913@163.com

Received November 23, 2018 / Accepted May 2, 2019

\begin{abstract}
Formyl peptide receptor-2 (FPR2) has been shown to promote various tumors, but its role in colorectal cancer (CRC) has not been clearly illuminated. The aim of this study was to investigate the effect of FPR2 interference on cell proliferation, migration, invasion, apoptosis, pro-angiogenesis of CRC cells, and also the mechanisms involved. Quantitative PCR assays were applied to assess the expression levels of FPR2 in CRC tissues. CRC cell line SW1116 was chosen to perform this study. We knocked down FPR2 gene by sh-RNA. Then, the cell proliferation was assayed by soft agar colony formation assay, the cell migration capacity was checked by wound healing assay, and cell invasion ability was detected by transwell assay. In addition, flow cytometric analysis was used to detect apoptosis, while endothelial tube formation assay was used to evaluate the effects of FPR2 on pro-angiogenesis in vitro. Tumorigenesis experiment in vivo was performed in nude mice. EMT-related proteins were studied by western blotting. Quantitative PCR demonstrated that FPR2 mRNA was highly expressed in the colorectal cancer tissues. SW1116 cells' capacities of proliferation, migration, invasion, anti-apoptosis and pro-angiogenesis were distinctly suppressed after silencing FPR2 in SW1116 by sh-RNA. Suppression FPR2 mRNA in SW1116 cells suppressed tumorigenicity in nude mice. The expression of proteins related to epithelial-mesenchymal transition (EMT) such as E-cadherin, N-cadherin, Snail, Slug and vimentin was changed after suppressing FPR2. In conclusion, our study demonstrated that FPR2 could promote CRC cells progression in vitro and in vivo that may relate to promoting EMT.
\end{abstract}

Key words: formyl peptide receptor-2, colorectal cancer, epithelial-mesenchymal transition

Colorectal cancer (CRC) is one of the most common malignancies worldwide, and its cancer-related mortality is the top three among all kinds of tumors [1]. Recurrence and metastasis are the main causes of death for CRC patients. Until recently, early diagnosis and surgical resection are still the most effective way for CRC. Lamentably, the available tumor biomarkers still lack sufficient sensitivity and specificity for the early diagnosis of CRC [2]. So a number of research groups including us are devoted to explore new ways to early diagnose or treat CRC.

Formylpeptide receptors-2 (FPR2), which plays an important role in inflammatory and immune responses, is one kind of G-protein coupled receptors [3]. FPR2 can be expressed under physiologic conditions in many normal cell types, such as microvascular endothelial cells, hepatocytes, neuroblastoma cells and fibroblasts $[4,5]$. However, lots of malignant tumors cells also express FPR2, which may promote tumor cell proliferation, invasion, pro-angiogenesis, etc. Human epithelial ovarian cancer cells and gastric cancer cells highly express FPR2, which predicts poor prognosis and promotes invasion and metastasis [6,7]. FPR2 can enhance laryngeal squamous cells growth and metastasis through paracrine way when it is activated by ANXA1 ligand [8]. However, there is little research about FPR2 and colorectal cancer until now. So we attempted to study the relationship between FPR2 and colorectal cancer.

We used quantitative PCR (qPCR) to detect the expression of FPR2 in the colorectal tumor tissues. Then, the FPR2 in SW1116 was knocked down by sh-RNA, while in the negative control group, cells were transfected by sh-RNA targeting nonhomologous gene. Subsequently, we studied the role of FPR2 in SW1116 cells by observing the proliferation, migration, invasion, apoptosis and pro-angiogenesis ability of each group of CRC cells.

\section{Materials and methods}

Patients and samples. We collected the patient's tumor tissues and adjacent normal mucosa of 50 cases, which were definitely diagnosed with $\mathrm{CRC}$ and resected by surgery at 
Ruijin Hospital, Shanghai Jiao Tong University School of Medicine from 2012 to 2015. These patients never received chemotherapy or radiotherapy before surgery.

Cell line and culture conditions. Seven human CRC cell lines (SW1116, HCT116, LoVo, SW620, RKO, Ht29 and Caco2) were purchased from the American Type Culture Collection (ATCC, Manassas, VA, United States) and cultured by Leibovitz's L-15 with $10 \%$ fetal bovine serum (FBS) (Gibco, Waltham, MA, USA). Cells were incubated with $5 \% \mathrm{CO}_{2}$ at $37^{\circ} \mathrm{C}$.

Quantitative PCR. FPR-2 primers were used as described [7]: FPR2 forward, 5'-AGTCTGCTGGCTACACTGTTC-3'; reverse, 5'-TGGTAATGTGGCCGTGAAAGA-3'. TRIzol reagent (Invitrogen, Carlsbad, CA, USA) was applied to extract total RNA. And reverse transcription kit (Invitrogen, Carlsbad, CA, USA) was used to perform reverse transcription. Quantitative PCR was performed using SYBR Green PCR Master Mix (Applied Biosystems, Warrington, UK) according to the manufacturer's protocol. GAPDH was measured as control.

Western blotting analysis. Protein from CRC cell line was extracted by RIPA Lysis Buffer (Beyotime Biotechnology, China) including 1\% PMSF (protease inhibitor, Thermo, USA). The protein concentration was determined by bicinchoninic acid (BCA) protein assay using BCA Protein Assay Kit (Thermo, USA). The samples and reagent were subsequently loaded into an electrophoresis chamber and separated by electrophoresis. Then, protein was transferred to the nitrocellulose by a western blot transfer apparatus (Bio-Rad Laboratories, USA) according to the standard protocol. The following antibodies were used to detect the protein expression respectively: rabbit polyclonal anti-FPR2 antibody (Abcam, UK, 1:5000); Epithelial-Mesenchymal Transition (EMT) Antibody Sampler Kit (including E-cad, N-cad, Slug, and others, Cell Signaling, USA, 1:1000); Rabbit polyclonal anti-GAPDH antibody (Cell Signaling, USA, 1:1000). Immunoblot images were scanned using an Odyssey Infrared fluorescence scanner (Lincoln, NE, USA).

Vector construction and transfection. The sh-RNA with plasmid targeting FPR2 was purchased from GenePharma (Shanghai, China). The shRNA sequences, which referred to Xiaohui Xie's research were as follows: ccggGGCCAAGACT TCCGAGAGAGActcgagTCTCTCTCGGAAGTCTTGGCCtttttg [7]. SW1116 cells were seeded onto 24 -well plates at density of $1 \times 10^{5}$ cells per well before transfection. Then the cells in experimental group were transfected by sh-RNA with plasmid targeting FPR2 mediated with Lipofectamine $2000^{\mathrm{rm}}$ until cell density was around $90 \%$. In negative control group, cells were transfected by sh-RNA with plasmid targeting nonhomologous gene in the same way. Total RNA and protein of cells were extracted $48 \mathrm{~h}$ later. Finally, quantitative PCR and western blotting techniques were applied to detect the shRNA inhibition efficiency.

Soft agar colony formation assay. sh-RNA targeting FPR2/nonhomologous gene transfected SW1116 cells were resuspended with $0.3 \%$ soft agar (Sigma, MO, USA) in Leibovitz's L-15 containing $10 \%$ FBS in six-well plates $\left(1 \times 10^{3}\right.$ cells per well). And then they were layered on $0.6 \%$ solidified agar in Leibovitz's L-15 with 10\% FBS. The plates were stained with crystal violet for $1 \mathrm{~h}$ after they were incubated with $5 \%$ $\mathrm{CO}_{2}$ at $37^{\circ} \mathrm{C}$ for 2 weeks. Colonies were not counted unless containing more than 50 cells.

Wound-healing assay. Cells $\left(1 \times 10^{5} /\right.$ well $)$ were seeded on 24 -well plates and grown to reach $90 \%$ confluence. Then scratches were uniformly made on the cells by a small pipette tip after attachment. Finally, the healing of scratches was examined under a microscope after the cells were incubated at $37^{\circ} \mathrm{C}$ in $5 \% \mathrm{CO}_{2}$ for another $36 \mathrm{~h}$.

Cell invasion assay. In vitro invasion capabilities were measured by transwell chambers (Corning, NY, USA). Each chamber was coated with $50 \mu$ l Matrigel (1:8 dilution; BD Biosciences, San Jose, CA, USA) on the upper compartment before cell seeding. When the glue solidified after 12 hours, each group of cells $\left(5 \times 10^{4}\right)$ was seeded in every upper chamber with serum-free medium. Cell culture medium containing 20\% FBS was added in the lower chambers. After incubating for 24/48 hours, cells were fixed by methanol (100\%) and stained with crystal violet $(1 \%)$ at $37^{\circ} \mathrm{C}$ for 30 min. Cells fixed on the lower side of the membranes were counted under the microscope.

Flow cytometric analysis for apoptosis. $3.3 \times 10^{5}$ SW 1116 cells per well were seeded into six-well plates without any antibiotics. 24 hours later, the cells were transfected with shRNA to FPR2 or scrambled sh-RNA. After 48 hours of transfection, two groups of cells were diluted and stained with AnnexinV/PI double staining kit (BD Biosciences, USA) according to the protocol. Finally, a FACScan (Beckman Instruments, Fullerton, CA, USA) was applied to detect the apoptotic cells.

Tube formation assay. The 48-well plate was coated with Matrigel uniformly at $100 \mu \mathrm{l} /$ well and incubated for 30 minutes until polymerizing. Human umbilical vein endothelial cells (HUVEC) $\left(5 \times 10^{4}\right.$ cells/well) were plated on the Matrigel with the culture medium from SW1116 and incubated for $5 \mathrm{~h}$ at $37^{\circ} \mathrm{C}, 5 \% \mathrm{CO}_{2}$.

Tumorigenesis experiment in vivo. 4 weeks old of male nude mice (BALB/C) were purchased from Shanghai Laboratory Animal Center of Chinese Academy of Sciences (Shanghai, China) and kept in a strict sterile laboratory of our hospital. We selected 20 nude mice in good condition and randomly divided them into 2 groups equally (Group 1 and Group 2). Mice of Group 1 were subcutaneously injected with $100 \mu \mathrm{l}$ SW1116 cells (conducted with sh-FPR2, $1 \times 10^{6}$ cells) into the right flank, while mice of Group 2 were injected with identical dose of SW 1116 cells (conducted with sh-NC, $1 \times 10^{6}$ cells) in the identical location to construct the tumor-bearing model. Mice were sacrificed after 4 weeks and the tumors were resected and measured. Tumor volume $\left(\mathrm{mm}^{3}\right)$ was calculated by a reduced formula: $0.5 \mathrm{x}^{2} \mathrm{y}$ ( $\mathrm{x}$ means the shorter diameter and y means the longer diameter). We 
chose another 20 nude mice and performed the investigation of their overall survival. These 20 mice were grouped Group I-II and processed as the same as the mice in Group 1-2. All the animal trials were performed with the approval and supervision by the Animal Ethics Committee of our unit.

Statistical analysis. Statistical analysis was performed using SAS 8.0 (SAS Institute Inc., Cary, NC, United States). All the data were expressed as mean $\pm \mathrm{SD}$, and all the assays were repeated more than three times. Wilcoxon MatchedPairs Signed-Ranks Test was applied to analyze the results of quantitative PCR, which demonstrated the relative expression value of FPR2 mRNA in tissues. Rank sum test was applied to analyze soft agar colony formation assay, woundhealing assay, invasion assay, cell apoptosis assay, endothelial tube formation assay and nude mouse tumorigenicity assay. Overall survival was compared by the log-rank test. A p-value $<0.05$ was considered statistically significant.

\section{Results}

High expression of FPR2 in CRC tissues. We took 50 cases of CRC tissue and normal tissue adjacent to tumor to explore the difference of FPR2 mRNA expression. Quantitative PCR results indicated that FPR2 mRNA expression in tumor tissues was significantly higher than normal tissues $(\mathrm{p}<0.05$, Figure $1 \mathrm{~A})$. The expression of FPR2 in seven CRC cell lines was detected by quantitative PCR. Results show that FPR2 is highly expressed in SW1116 cell line (Figure 1B). Because of the good state of the SW1116 cell line, we chose it for our research.

Silencing of FPR2 decreased proliferation, migration and invasion. Quantitative PCR analysis and western blotting results demonstrated that the expression of FPR2 (mRNA and protein) was reduced by the shRNA distinctly (Figure 2A, B). Analysis of colony formation assay revealed that the proliferation of SW1116 cells was significantly weakened when FPR2 was downregulated (Figure 2C), obviously less colonies in sh-FPR2 group than in the control group (57.4 \pm 14.40 vs. $122.2 \pm 17.12, \mathrm{p}<0.05)$. Also wound-healing assay demonstrated that the cells in the experimental group migrated at shorter distances than the control group in $36 \mathrm{~h}(0.8 \pm 0.25 \mathrm{vs}$. $1.7 \pm 0.19$ ). It means that cell migration ability of SW 1116 cells was obviously weakened when FPR2 was downregulated by shRNA. The difference was statistically significant $(\mathrm{p}<0.05)$ (Figure 2D). Transwell assay was used to verify the effect of FPR2 on SW1116 cell invasion in vitro. The results showed that the number of invaded cells was abated after SW1116 was treated with shRNA to FPR2. The difference showed statistically significant ( $\mathrm{p}<0.05$, Figure $2 \mathrm{E})$.

FPR2 can inhibit apoptosis of SW1116 cells. Cell apoptosis analysis was carried out to detect the apoptosis of SW1116 cells. Results showed SW1116 cells induced a distinct decrease in the apoptosis rate after downregulating FPR2 $(24.9 \pm 4.58$ vs. $6.8 \pm 0.89, \mathrm{p}<0.05)$ (Figure $3 \mathrm{~A})$. Therefore, FPR2 can obviously inhibit apoptosis of tumor cells.

FPR2 promoted the angiogenesis of SW1116 cells in vitro. We further investigated the effects of FPR2 on the pro-angiogenesis capabilities of CRC cells in vitro. When the HUVEC cells were stimulated by the culture medium of SW1116 cells with silenced FPR2, the number of tube formation was observably lower than in the negative control $(\mathrm{p}<0.05$, Figure 3B).

FPR2 enhanced tumorigenesis in vivo. The growth rate of nude mice tumors in sh-FPR2 group was significantly
A

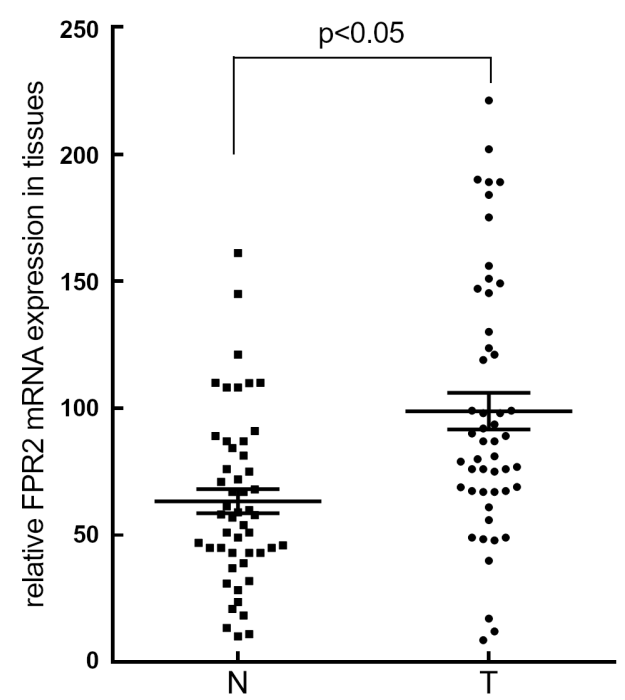

B

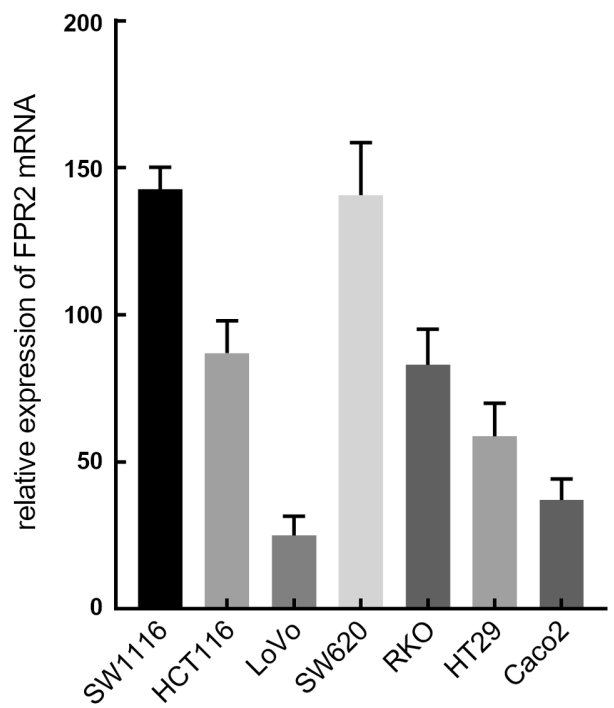

Figure 1. A) Relative expression of FPR2 mRNA in tumor samples (T) was significantly increased in comparison to normal (N) tissues. B) The expression of FPR2 mRNA in seven CRC cell lines. 
A

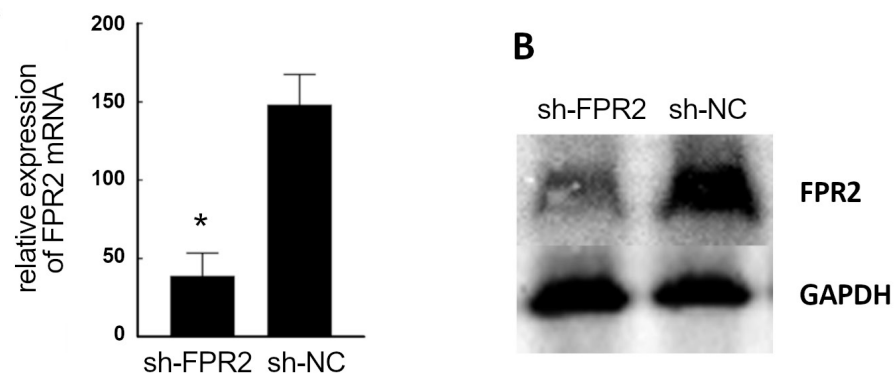

C
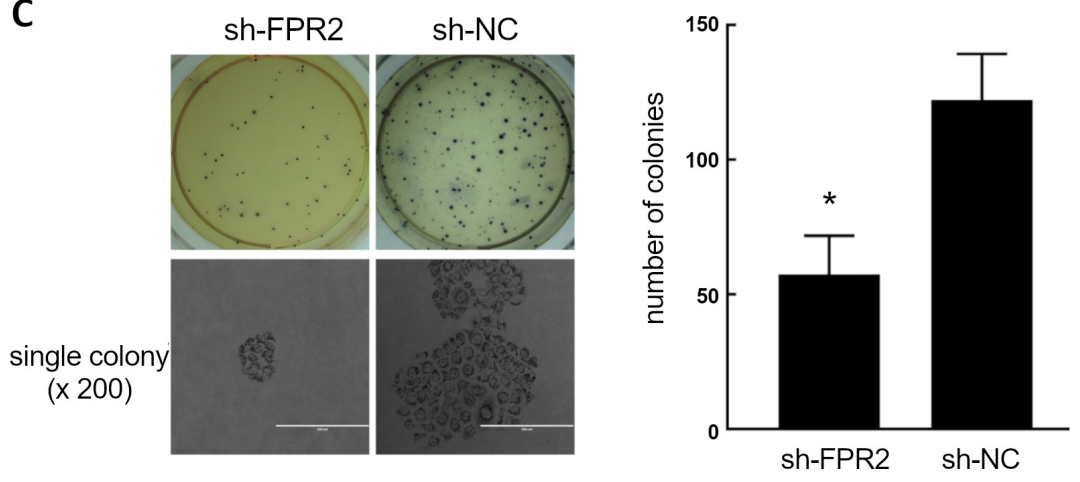

D
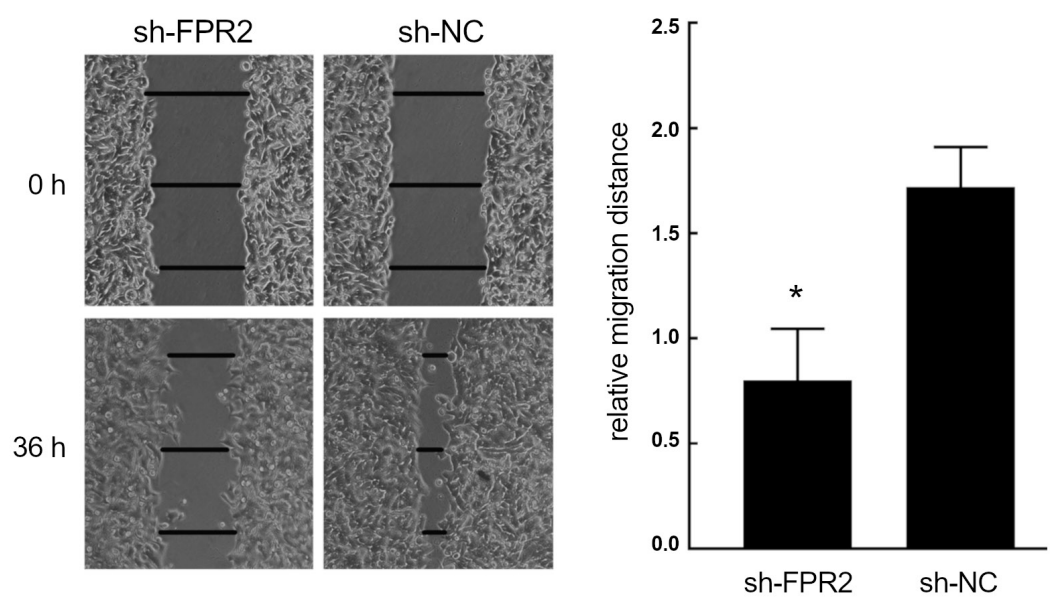

E
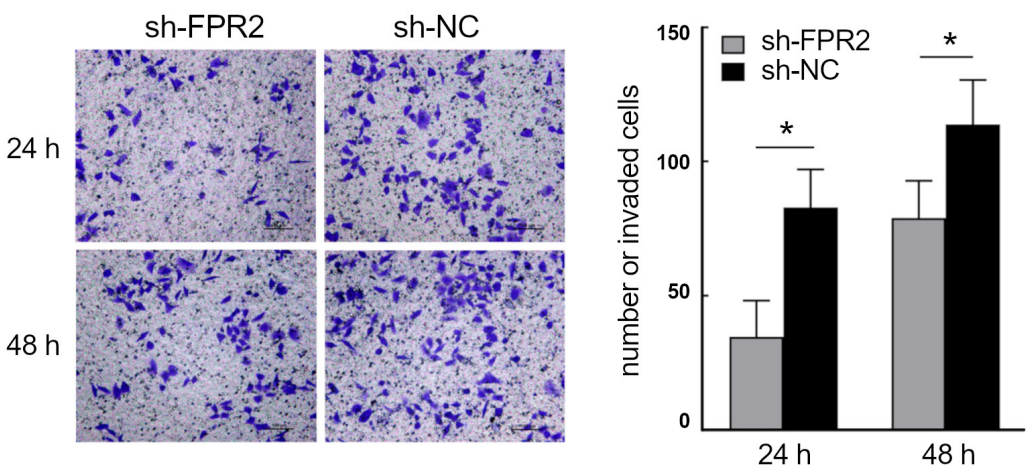

Figure 2. The effectiveness of FPR2 silencing on A) FPR2 mRNA and B) protein levels, C) soft agar colony formation, D) migration and E) invasion of SW 1116 cells compared to sh-NC control. ${ }^{*}$ : means $\mathbf{p}<0.05$. 
$\mathbf{A}$
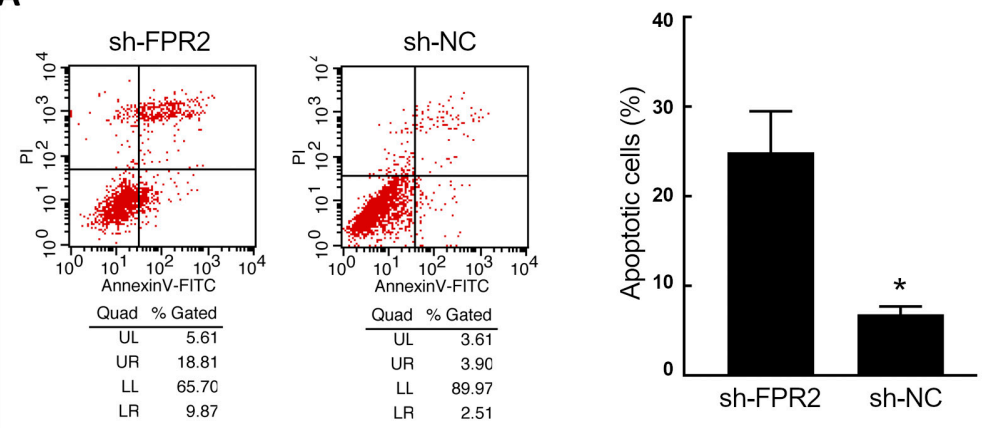

B
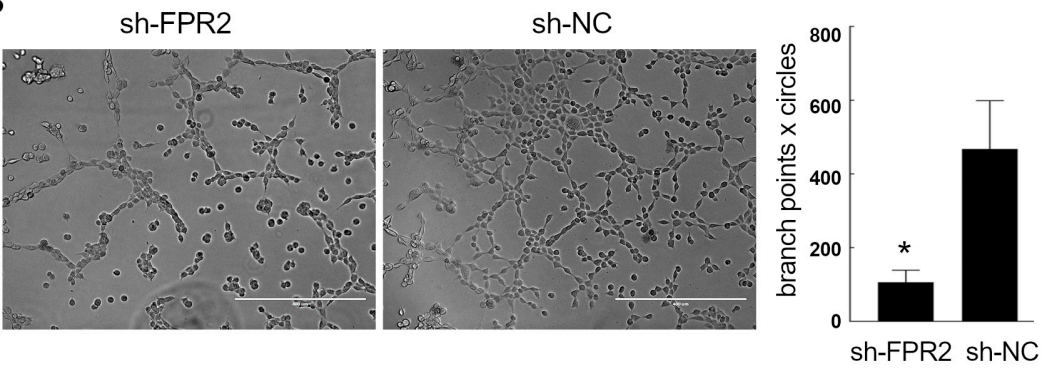

Figure 3. A) The apoptosis rate of sh-FPR2 group was significantly higher compared to control group ( $<<0.05)$, B) Tube formation using HUVEC cells demonstrated (expressed as number of branch points $\mathrm{x}$ number of branches per field) a decrease in sh-FPR2 in comparison to control sh-NC group. ${ }^{\star}$ means $\mathrm{p}<0.05$.

A

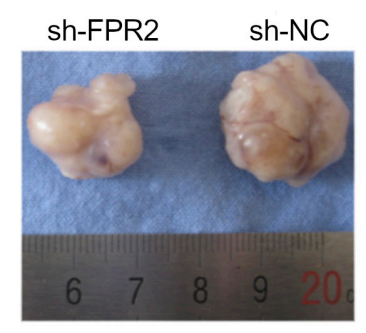

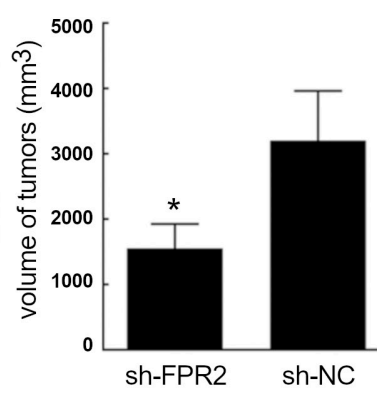

B

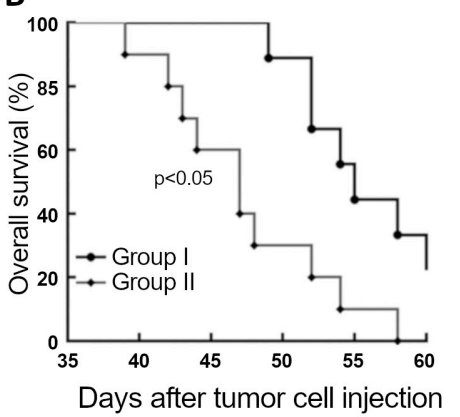

Figure 4. The tumor volumes in Group 1 (mice injected with sh-FPR2 transfected SW1116 cells) were significantly smaller than in the control Group 2 mice; the overall survival of Group I and Group II shows statistically significant difference. ${ }^{\star}$ means $\mathbf{p}<0.05$.

slower than that of control group. The results showed that the tumor volumes of mice in Group 1 were significantly smaller than that of Group $2(1550 \pm 376.95$ vs. $3201 \pm 762.53, \mathrm{p}<0.05$, Figure $4 \mathrm{~A})$. Furthermore, the overall survival of nude mice in the Group I was significantly longer than that in the Group II (Figure 4B).

FPR2 enhanced migration and invasion of SW1116 cells by promoting EMT. It is well known that epithelial-mesenchymal transition (EMT) of tumor cells is closely related to tumor metastasis. To investigate whether FPR2 enhanced SW1116 cells migration and invasion by promoting EMT process, we applied an EMT Antibody Sampler Kit (Cell Signaling Technology, USA). Results demonstrated that when FPR2 of SW1116 was downregulated, the expression of E-cadherin gene increased. However, the expression of $\mathrm{N}$-cadherin, slug, snail and vimentin decreased significantly (Figure 5).

\section{Discussion}

According to current researches, FPR2 can promote the development of numerous solid tumors, such as ovarian cancer, gastric cancer, lung cancer, laryngeal squamous cell cancer [6-11]. But the role of FPR2 in CRC is rarely reported. We conjecture that FPR2 can also promote the development of CRC.

FPR2 is highly expressed in various tumor tissues such as ovarian cancer and gastric cancer $[6,7]$. In our study, quanti- 

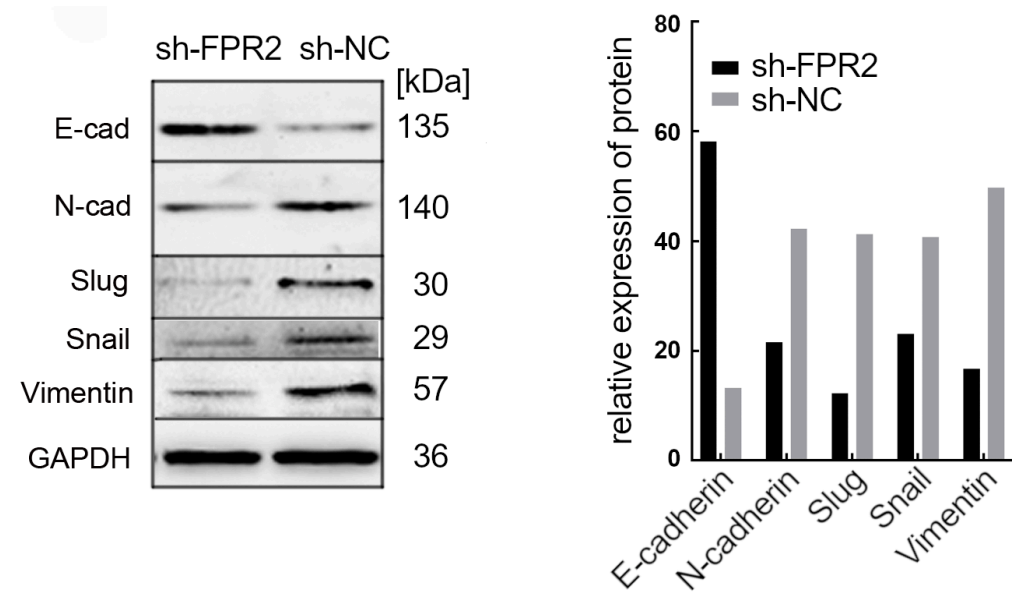

Figure 5. Changing of EMT-related proteins expression in FPR2 silenced SW1116 cells.

tative PCR results showed that FPR2 was highly expressed in CRC tissues, but downregulated or absent in normal colorectal tissues. It suggests that FPR2 may play a role in promoting colorectal cancer. CRC cell line SW1116 was screened to perform cytology experiments due to its high expression of FPR2 and good condition. FPR2 has been reported to promote the proliferation of laryngeal squamous cell carcinoma and breast cancer $[8,10]$. Our study also demonstrated that FPR2 promoted tumor proliferation of CRC cells by soft agar colony formation assay. FPR2 can promote migration and invasion of gastric cancer, ovarian cancer and glioma $[6,7,11]$, while our research results (wound healing assay and cell invasion assay) also revealed that FPR2 could enhance the migration and invasion ability of CRC cells. Xie et al. revealed that FPR2 could promote tumor angiogenesis in ovarian cancer [7]. In our study, tube formation assay also demonstrated that FPR2 could promote tumor angiogenesis in CRC cells. In addition, our study found that FPR2 could inhibit CRC cell apoptosis by flow cytometric analysis. Therefore, our study shows that FPR2 can promote the proliferation, migration, invasion, pro-angiogenesis and anti-apoptosis of CRC cells in vitro.

To further confirm the tumor-promoting effect of FPR2, in vivo experiments are also essential. Recent studies have shown that FPR2 promotes gastric and breast cancer progression in vivo $[6,10]$. In our study, nude mice assay demonstrated that FPR2 could promote the growth of CRC cells in vivo. Moreover, higher expression of FPR2 predicted shorter survival. In conclusion, our study revealed that FPR2 promoted CRC cells in vitro and in vivo. It indicates that FPR2 may play an important role in promoting the growth and metastasis of CRC.

It has been clear that EMT process promoted tumor migration and invasion [12]. And reports showed that FPR2 enhances gastric cancer and lung cancer progression by promoting EMT process $[6,13]$. Our research also demon- strated that FPR2 could downregulate E-cadherin and upregulate $\mathrm{N}$-cadherin, Snail and Vimentin obviously. It suggested that FPR2 could promote EMT process in CRC cells. Whether FPR2 can promote other CRC cell lines and whether there are any other mechanisms that FPR2 promoted CRC will be explored in our further researches.

In conclusion, FPR2 can promote colorectal cancer progression in vitro and in vivo. And it may relate to promoting EMT.

Acknowledgments: This study was funded by grants from National Natural Science Foundation of China (81201625).

\section{References}

[1] SIEGEL RL, MILLER KD, JEMAL A. Cancer Statistics. CA Cancer J Clin 2017; 67: 7-30. https://doi.org/10.3322/ caac. 21387

[2] MAIDA M, MACALUSO FS, IANIRO G, MANGIOLA F, SINAGRA E et al. Screening of colorectal cancer: Present and future. Expert Rev Anticancer Ther 2017; 17: 1131-1146. https://doi.org/10.1080/14737140.2017.1392243

[3] LI Y, YE D. Molecular biology for formyl peptide receptors in human diseases. J Mol Med (Berl) 2013; 91: 781-789. https:// doi.org/10.1007/s00109-013-1005-5

[4] MIGEOTTE I, COMMUNI D, PARMENTIER M. Formyl peptide receptors: a promiscuous subfamily of $\mathrm{G}$ proteincoupled receptors controlling immune responses. Cytokine Growth Factor Rev 2006; 17: 501-519. https://doi. org/10.1016/j.cytogfr.2006.09.009

[5] YE RD, BOULAY F, WANG JM, DAHLGREN C, GERARD $\mathrm{C}$ et al. International Union of Basic and Clinical Pharmacology LXXIII: Nomenclature for the formyl peptide receptor (FPR) family. Pharmacol Rev 2009; 61: 119-161. https://doi. org/10.1124/pr.109.001578 
[6] HOU XL, JI CD, TANG J, WANG YX, XIANG DF et al. FPR2 promotes invasion and metastasis of gastric cancer cells and predicts the prognosis of patients. Sci Rep 2017; 7: 3153. https://doi.org/10.1038/s41598-017-03368-7

[7] XIE X, YANG M, DING Y, YU L, CHEN J. Formyl peptide receptor 2 expression predicts poor prognosis and promotes invasion and metastasis in epithelial ovarian cancer. Oncol Rep 2017; 38: 3297-3308. https://doi.org/10.3892/ or.2017.6034

[8] GASTARDELO TS, CUNHA BR, RAPOSO LS, MANIGLIA JV, CURY PM et al. Inflammation and cancer: role of annexin A1 and FPR2/ALX in proliferation and metastasis in human laryngeal squamous cell carcinoma. PLoS One 2014; 9: e111317. https://doi.org/10.1371/journal.pone.0111317

[9] CATTANEO F, IACCIO A, GUERRA G, MONTAGNANI S, AMMENDOLA R. NADPH-oxidase-dependent reactive oxygen species mediate EGFR transactivation by FPRL1 in WKYMVm-stimulated human lung cancer cells. Free Radic Biol Med 2011; 51: 1126-1136. https://doi.org/10.1016/j.freeradbiomed.2011.05.040
[10] MORAES LA, KAR S, FOO SL, GU T, TOH YQ et al. Annexin-A1 enhances breast cancer growth and migration by promoting alternative macrophage polarization in the tumour microenvironment. Sci Rep 2017; 7: 17925. https://doi. org/10.1038/s41598-017-17622-5

[11] LIU L, LI X, SHI J, LI L, WANG J et al. [Effects of FPR2 gene silencing on the proliferation, migration and invasion of human glioma U87 cells]. Zhonghua Zhong Liu Za Zhi 2018; 40: 659-666. https://doi.org/10.3760/cma.j.is sn.0253-3766.2018.09.004

[12] THIERY, JP. Epithelial-mesenchymal transitions in tumor progression. Nat Rev Cancer 2002; 2: 442-454. https://doi. org/10.1038/nrc822

[13] Lee HJ1, Park MK, Lee EJ, Lee CH. Resolvin D1 inhibits TGF- $\beta 1$-induced epithelial mesenchymal transition of A549 lung cancer cells via lipoxin A4 receptor/formyl peptide receptor 2 and GPR32. Int J Biochem Cell Biol 2013; 45: 28012807. https://doi.org/10.1016/j.biocel.2013.09.018 